\title{
Skatologismen in aggressiven Sprechakten
}

\section{Einleitung}

Im Anschluss an das Konzept der >expressiven Sprechakte ‘ von John R. Searle, ${ }^{1}$ die Gefühle und Einstellungen der sie verwendenden Person zum Ausdruck bringen, bezeichne ich Sprechakte, die primär zum Abreagieren negativer Emotionen und/oder zur Beleidigung eingesetzt werden, als aggressive Sprechakte. Zu den aggressiven Sprechakten zähle ich: Beschimpfung, Fluch, Verwünschung, Drohung und aggressive Aufforderung. Im breiten Sinne gehören dazu auch beleidigende Äußerungen und Vergleiche sowie Emotions- und Situationsthematisierungen, die von Befragten auch als Äußerungen verbaler Aggression identifiziert werden.

Skatologische oder koprologische Lexik (von griech. skor, skatos bzw. kopros - >Stuhl«) umfasst Wörter, die sich im breiten Sinne auf Ausscheidungsprodukte des menschlichen Körpers (Stuhl, Harn, Nasenschleim, Sperma, Speichel usw.) und entsprechende Vorgänge beziehen.
Im Beitrag werden strukturell-semantische und pragmatische Besonderheiten der Skatologismen im Deutschen, ihr Gebrauch in aggressiven Sprechakten und ihr Funktionsspektrum vorgestellt. Die empirische Grundlage bilden schriftliche (538 Personen) und mündliche (72 Personen) Umfragen (insg. 610 Wienerinnen und Wiener zwischen 14 und 90 aus verschiedenen sozialen Gruppen) im Rahmen zweier FWF-Forschungsprojekte (2006-2008 und 2012-2017), sowie literarische Texte und Zeitungsberichte. 
Im engen Sinne wird darunter diejenige Lexik verstanden, die sich auf den fäkal-analen Bereich bezieht und auf die sich mein Beitrag beschränkt. Als skatologische Kernlexeme betrachte ich dabei >scheißen`, >Scheiße ( sowie mildere Varianten $>$ Dreck $\iota$, , Mist $\iota$, ,Kacke $\iota)$ und $>$ Arsch $\iota$, die zur Bildung einer Reihe strukturell-abgeleiteter (formeller) oder semantisch abgeleiteter Pejorativa sowie idiomatischer Wendungen dienen und in aggressiven Sprechakten anzutreffen sind.

Die Liste skatologischer Wörter und Wendungen ist in vielen Sprachen lang und kann mit der Abneigung gegen den Schmutz erklärt werden, die vielen Kulturen eigen ist und mit uralten Tabus zusammenhängt. Dennoch lassen sich Kulturen und dementsprechend Sprachen hervorheben, in denen dem Skatologischen eine besondere Rolle zukommt. Hinsichtlich der Verankerung des Schimpfvokabulars zählt das Deutsche zur >fäkal-analen Schimpfkultur ${ }^{2}{ }^{2}$ Sprach- bzw. Kulturwissenschaftlerinnen und -wissenschaftler, die sich mit dem pejorativen Vokabular beschäftigen, betonen, dass es in keiner europäischen Sprache so viele Wörter und Wendungen aus dem fäkal-analen Bereich gibt, wie im Deutschen. ${ }^{3}$ Das Dominieren im pejorativen Vokabular der skatologischen Lexik über der sexuellen zeigt sich auch in den angeführten Pejorativa, die ich per Fragebogen im Rahmen schriftlicher Umfragen zum Thema >verbale Aggression` in Wien in zwei FWF- Forschungsprojekten (Lise Meitner-Programm 2006-2008 und Elise Richter-Programm 2012-2017) gewonnen habe. ${ }^{4}$ Zwei von vielen aussagekräftigen Beispielen seien hier angeführt: ${ }^{5}$

>Arschloch, geh scheißen, Schastrommel, g'schissene Sau

,Scheiße, Scheißdreck, Kacke, Kackdreck, Scheißkackendreck, fick dich, verdammt, verdammte Scheiße, Fickendreck, nicht cool, Trottl, Arschloch, Oasch, verfickt, verficktes ${ }^{6}$ (irgendsowas) verckackt, fuckdreck, Hurenckackendreck, Fotze, krasses Arschloch,

Deppert, Drecks Kotz

2 Aman: Die klügsten Beschimpfungen, S. 34; Stavyc'ka: Украӥнська мова без табу, S. 33.

3 Aman: Die klügsten Beschimpfungen, S. 34; Dundes: Sie mich auch!, S. 19; Gauger: Das Feuchte und das Schmutzige, S. 47ff. u. 57ff.; Zhelvis: Поле брани, S. 227f.; Kiener: Das Wort als Waffe, S. 144; Stavyc'ka: Украӥнська мова без табу, S. 33.

4 Die Befragten wurden nach dem persönlichen Gebrauch von Pejorativa sowohl in aggressiven Sprachakten als auch in neutralen Kontexten gefragt.

5 Ein Vergleich der von mir durchgeführten schriftlichen und mündlichen Umfragen zeigt, dass die pejorativen Wörter und Wendungen in der gesprochenen Sprache in dialektaler Form erscheinen, während sich die Befragten bei ihrer schriftlichen Wiedergabe der Standardsprache bedienen. - Alle Zitate aus den Umfragen werden in der Form angeführt, wie sie in den Fragebögen oder bei den Interviews vorkommen und durchgehend mit >einfacher Anführung ‘ gekennzeichnet. Sind mehrere Beispiele gemeinsam durch einfache Anführung gerahmt, dann stammen sie alle von ein und derselben Person.

6 Die Striche stehen für die Angabe eines beliebigen Wortes. 
Es kommt auch zur Kombination der fäkal-analen Sphäre und der sexuellen Sphäre - entweder in Teilen des zusammengesetzten Lexems, in Verbindung mit einem Attribut oder mit einem anderen aggressiven Sprechakt:

,Drecksschwanz, Drecksschwuchtl, Drecksschlample, Drecksfotzn, Dreckshur (A so a oage Dreckshur), verficktes Dreck, Fickendreck, fuckdreck, Hurenckackendreck, du verficktes Scheißding, geficktes/verficktes/verhurtes Arschloch, Oaschfotzn, Oaschhur

,Fick dich, Scheißoaschloch!

Die starke Dominanz des skatologischen Elements gegenüber dem sexuellen im Deutschen wird als spezifische Protestform (als verbaler Tabubruch) gegen die von der Gesellschaft vorgeschriebenen Regeln, Sauberkeit und Ordnung einhalten zu müssen, sowie gegen die starke Reglementierung des Lebens in den deutschsprachigen Ländern betrachtet. ${ }^{7}$ Aus dieser Perspektive wird auch die Beliebtheit der Fäkalsprache in fäkalen Orgien im Rahmen der Studentenrevolte und in der Kindersprache nachvollziehbar: Protest gegen den gesellschaftlichen oder elterlichen Zwang, Freude am Übertreten von Verboten und am Provozieren.

\section{Skatologische Lexik im Deutschen}

Die Skatologismen können in direkter wie in übertragener Bedeutung verwendet werden, was am Beispiel von `Scheiße deutlich wird:

- direkte Bedeutung: >Ich bin in die Scheiße getreten.^

- übertragene Bedeutung:

a) als universales Schimpfwort: `Du falsches Stück Scheiße! So ein Stück Scheiße!

b) als Fluch in einer ärgerlichen Situation: ১Scheiße!

Im weiten Sinne werden skatologische Schimpfwörter auch als Vulgarismen bezeichnet. Im engen Sinne kann zwischen diesen Begriffen eine Trennlinie gezogen werden, wobei als distinktives Merkmal der Bezug des Schimpfwortes auf den Menschen bzw. auf das Tier oder den Gegenstand gilt. Die Vulgarismen sind dagegen stilistisch unterlegte Bezeichnungen von Körperteilen (Arsch - »Einen Arsch hatte die wie ein Pinzgauer runterfallen konnte man bei der nicht! « $\left.{ }^{8}\right)$ oder physiologischen Prozessen

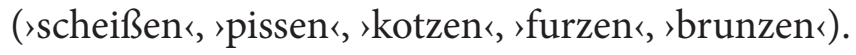

7 Dundes: Sie mich auch!, S. 74; Zhelvis: Поле брани, S. 227; Stavyc'ka: Українська мова без maбy, S. 33; Kiener: Das Wort als Waffe, S. 145.

8 Hinterberger: Mundl, S. 163. 
In übertragener Bedeutung treten die Vulgarismen auch in vulgären Äußerungen, die die Form fester Redewendungen haben und entweder mit dem Ziel eingesetzt werden, der Sprache besonderen Ausdruck und Intensität zu verleihen (intentionaler Gebrauch) oder zum Grundwortschatz der Personen, die sie gebrauchen, gehören - wie in den unten angeführten Aussagen von Prostituierten und Zuhältern, ${ }^{9}$ wenn Vulgarismen unbewusst in neutralen bzw. emotionell schwach geladenen Situationen zur Bezeichnung von Vorgängen ( $>$ in den Arsch pudern $`$ ), Körperteilen ( $>$ Arsch $`$ ) oder Situationen und Sachverhalten (`Arsch-Jugend ) verwendet werden:

"dann haut sie ihn wieder in den Arsch $\aleph^{10}$

"Ich bin geborener Wiener und habe eine Arsch-Jugend gehabt «"

»und solche, die gerne griechisch machen, die Arschpuderer ${ }^{12}$

Abgesehen von diesen Milieus zeigt das Untersuchungskorpus für diesen Beitrag, dass sich der Gebrauch von vulgären Äußerungen trotz vertretener Hypothesen, die Personen aus niedrigen sozialen Schichten eine derbere Sprache zusprechen, ${ }^{13}$ schichtspezifisch homogen zeigt. Ein Beispiel schichtübergreifenden Gebrauchs von Vulgarismen stellt die Äußerung eines Dompfarrers dar: »Was die Anzahl der Mitglieder betrifft, ist die Lage relativ beschissen. « ${ }^{14}$ Der Geistliche bedient sich eines Vulgarismus, um seine Aussage zu verstärken und dadurch die Ernsthaftigkeit kirchlicher Probleme zu betonen. Die Wirkung intentional gebrauchter vulgärer Äußerungen seitens prominenter Persönlichkeiten hebt auch Kiener hervor: »Die Fäkalsprache wirkt oft gerade im Munde Prominenter überraschend und - schlagend $\aleph^{15}$

Vulgarismen treten auch in idiomatischen Redewendungen in Form von Emotionsthematisierungen auf ( $>$ Das geht / du gehst ma (schon so was) am Oasch ‘; >Du zehrst ma am Oasch «; >Das geht mir am Arsch vorbei $<$ ) oder sie können sich auf eine Situation, einen Sachverhalt oder einen Ort beziehen:

>Das war ein Griff in den Arsch

>die spielen einen Scheiß zusammen (beim Fußballspiel)

>das ist am Arsch der Welt<

,j-m am Schädel scheißen<

, $j$ - $n$ bescheißen/anscheißen

Girtler: Der Strich.

10 Ebd., S. 99.

11 Ebd., S. 110.

12 Ebd., S. 185.

13 Jay: Why We Curse; Stavyc'ka: Українська мова без табу.

14 Dompfarrer: "Kirche geht's beschissen «, "ÖSTERREICH« (14.11.2014), S. 8.

15 Kiener: Das Wort als Waffe, S. 145. 
>ich habe Scheiße gebaut

sin der Scheiße sitzen

>die Scheiße steht uns zum Hals

reinen Scheiß reden<

>des is a Schaaß/Lercherlschaaß

Vulgäre Äußerungen werden oft gebraucht, um die Realität abzuwerten, Angst zu bewältigen bzw. als Selbstanspornung in stressigen Situationen. ${ }^{16}$

»dass die Welt ein `Schas sei und sie deshalb auch `Schas $`$ machen ${ }^{17}$

"SCHEISS auf die WAHLEN! « (auf einem Aufkleber)

`Geh, scheiß drauf! Auf das wird geschissen!^

>Ach scheiß doch der Hund drauf!

`Scheiß da Hund aufs Feuerzeug, braucht a kan Benzin!

Im Weiteren wird das Augenmerk auf die Skatologismen im Bereich der formellen sowie der metaphorischen und metonymischen Pejorativa gelegt.

\subsection{Strukturell-abgeleitete (formelle) Pejorativa}

Im Bereich der strukturell-abgeleiteten Pejorativa sind Modelle mit Halbpräfixen (>Präfixoiden $`$ ) produktiv. Zu den skatologischen Halbpräfixen

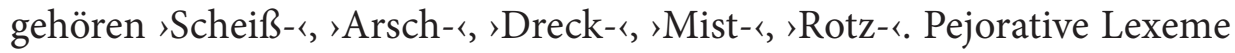
entstehen dabei nach folgenden Modellen:

a) Pejoratives Halbpräfix + neutrales Lexem. Nach diesem Modell werden neue pejorative Lexeme gebildet, wobei die Kombinationsmöglichkeiten und die Liste der gebildeten Pejorativa unbegrenzt ist (vgl. den Kommentar in einem Fragebogen: >Alles, was mich ärgert, wird mit der Vorsilbe Scheiß- verziert $`$ ):

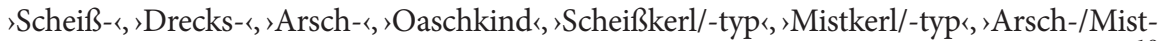

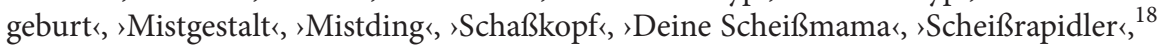

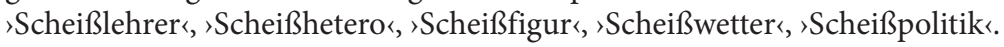

Dieses Modells bedient sich auch Wolfgang Bauer bei der Darstellung eines Streites in dem Theaterstück Magic Afternoon, wobei er auf die Freiheit bei der Bildung von Pejorativa hinweist:

[...] es kommt zu einer regelrechten Bücherschlacht, bei der beide langsam immer fröhlicher werden. Bevor sie werfen, schreien sie die Autoren der Bücher. Also z.B. ScheißDürrenmatt, Scheiß-Pinter, Scheiß-Albee, Scheiß-Walser, Scheiß-Grass, dann immer fröhlicher werdend: Scheiß-Ionesco, Scheiß-Beckett [...] (beide lachen schon) Jetzt eine abschließende Balgerei mit den Klassikern: Scheiß-Goethe, Scheiß-Schiller... etc. ${ }^{19}$ 
Durch die Verbindung mit dem Halbaffix >scheiß- $<$ werden die neutralen Eigenschaftswörter intensiviert (bspw. >scheißegal«, >scheißzuwider`, >arschkalt $\iota$, arschknapp ‘): »Holt Van der Bellen heute Wahlsieg? Es wird arschknapp! « ${ }^{20}$ Die positiven Eigenschaftswörter können dank der Verbindung mit dem Halbaffix 〉scheiß-〈 eine Bedeutungsverschlechterung erleben - wie bei `scheißfreundlich`, wenn die anfangs positive Eigenschaft durch das Übertreiben als negativ wahrgenommen wird.

Dieses Wortbildungsmodell ist auch bei der Bildung von Regional- und Nationalschelten sowie von Sachschelten produktiv:

- Scheiß- + Herkunft/Nationalität:

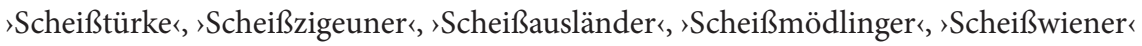

- Scheiß- (Oasch-, Drecks-) + Gegenstandsbezeichnung:

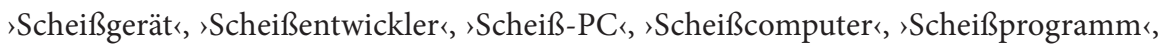

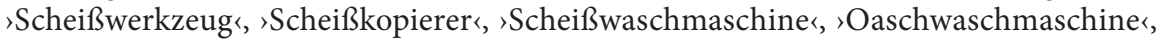

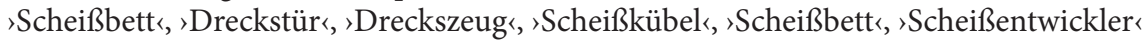

Der unbegrenzten Möglichkeiten zur Bildung von Sachschelten sind sich die Sprachträgerinnen und Sprachträger bewusst, wovon ihre metasprachlichen Kommentare zeugen (hier in Klammern), z.B.: >Scheißkastl (und andere Gegenstände nach dem Muster). Mit Hilfe dieses Halbpräfixes kann auch die schon existierende Sachschelte intensiviert werden: >Mist-/ Scheiß-/Drecksklumpert`.

b) Pejoratives Halbpräfix + pejoratives Lexem; das Halbpräfix verstärkt in diesem Fall das pejorative Lexem:

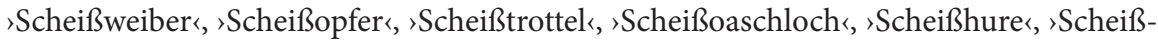

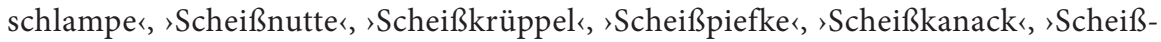

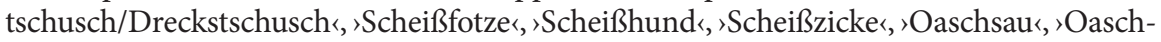

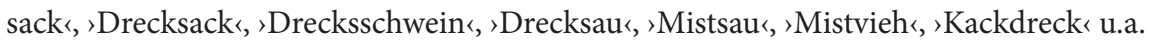

Auch die Kombination von zwei skatologischen Halbpräfixen kommt

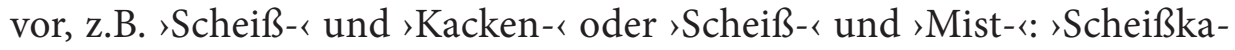
ckendreck $\iota$, Scheißmistgeburt .

\subsection{Metaphorische und metonymische Pejorativa}

Im Bereich der metaphorischen Pejorativa sind Skatologismen in zwei Gruppen vertreten: 
a) Fäkalien und Ausscheidungen (〉Mist «, >Miststück〈, >Misthaufen`,

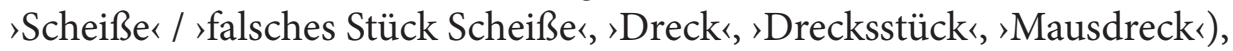
die in erweiterter Form auch als okkasionelle Genitiv- und Dativmetaphern ( Schas mit Schubumkehr = >Arschkriecher «; »Du Stuhlgang einer Hexe! « ${ }^{21}$ ) auftreten können. Kiener meint, dass sich diese Wendungen bei Zornausbrüchen selten einstellen, eher dort, "wo man Kraftausdrücke demonstriert und der Schimpfende auch rhetorisch zur Geltung kommen möchte«. ${ }^{22}$ Die von mir durchgeführten mündlichen und schriftlichen Umfragen bestätigen diese These: Im Affektzustand hat die schimpfende Person keine Zeit zum Erfinden phantasievoller Konstruktionen und greift deshalb auf häufige und einfache Wörter und Wendungen zurück.

b) Handlungen, von denen pejorative Personenbezeichnungen gebildet

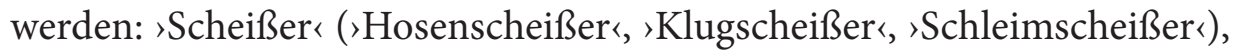

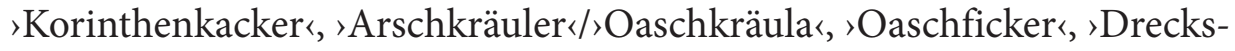
schleuder . Der Mechanismus dieses Vergleichstyps besteht darin, dass der Charakter (die Eigenschaft oder das Benehmen) des Adressaten / der Adressatin durch metaphorische Umbesetzung der Handlung wiedergegeben wird. Dieser Vergleichstyp ist im Deutschen sehr produktiv - nach dem beschriebenen Modell können immer neue spontane Pejorativa gebildet werden. Wörtliche Bedeutung einzelner Pejorativa dieser Gruppe ist oft

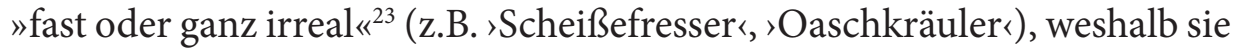
sich durch eine noch stärkere Bildhaftigkeit auszeichnen und seltener in realen Konfliktsituationen als in literarischen Texten zu finden sind: »Sie gemein verstrudelter Schweinescheißefresser ohne Schamgefühl! «; ${ }^{24}$ ॥hr Todesficker, ihr geilen Menschenaasfresser «. ${ }^{25}$

$\mathrm{Zu}$ den häufigen metonymischen Skatologismen zählen: ‘Arsch`, >Arschloch , 〉Oaschwarzn`, sowie erweiterte metonymische Konstruktionen: >Arsch mit Ohren`, >Arschgesicht mit Quastln`, ‘größter Oasch zwischen Scheibbs und Nebraskar.

Eine besondere Gruppe der Stilfigur pars pro toto bilden Bahuvrihi - metonymische Zusammensetzungen mit einem adjektivischen Bestim-

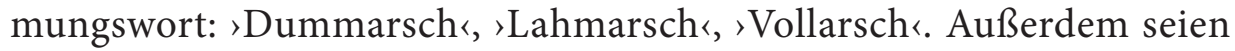
Zusammensetzungen erwähnt, deren erste Komponente ein Substantiv oder ein Verbalstamm ist: `Hurenarsch`, ,Saftarsch`.

22 Kiener: Das Wort als Waffe, S. 156.

23 Ebd., S. 148.

24 Schwab: Faust, S. 108.

25 Schwab: Übergewicht, unwichtig, S. 87. 


\section{Sprechakt `Beschimpfung` und seine Formen}

\subsection{Verbindungen mit Attributen}

Im Sprechakt 〉Beschimpfung` treten Skatologismen häufig in Verbindung mit Attributen auf. Dabei können skatologische Pejorativa, die über eine universale pejorative Bedeutung verfügen, durch das Attribut konkretisiert (`faules Arsch $`$ ) oder verstärkt werden (`verdammtes Arschloch ‘, >versautes Arschloch $\iota$, ,verdammter Scheißdreck ).

Skatologismen können auch in Form von konkretisierenden (`schasaugerter Fetzenschädel`) oder intensivierenden Attributen (`g’schissener Wixer , `arschgestopftes Schwein «, >arschgeficktes Hurenkind ) funktionieren.

Treten die Skatologismen in Verbindung mit skatologischen Attributen auf ('g’schissener Scheißdreck`, geschissenes/beschissenens Arschloch ‘; >stinkendes Arschloch ‘), können wir von `kopierenden Attributen $`$ reden. Wortverbindungen mit kopierenden Attributen zähle ich zu den Pleonasmen, da es hier um Verbindung von Wörtern gleicher oder ähnlicher Bedeutung geht.

Kennzeichnende Besonderheit des Sprechaktes `Beschimpfung ‘ besteht darin, dass das Attribut oft nachgestellt wird: `Orschloch, verbeschissenes!‘; ,Scheißdreck, verdammter! ‘; Oaschwarzn graupade!

Eine eigenständige Gruppe bilden zweigliedrige Aussagen, die aus einem pejorativen Attribut und einem neutralen Lexem, das den realen AdressatInnenstatus bezeichnet, bestehen. In diesen Aussagen generiert das Attribut die Pejorativität der ganzen Aussage: >beschissener Mensch`, sarschgeficktes Kind . Dieses Modell ist auch bei der Bildung von pejorativen Wortverbindungen produktiv, die sich auf Sachverhalte und insbesondere - auf Gegenstände - beziehen: ১beschissene(s)/verschissene(s) Situation/Wetterı, ‘beschissenes Kabel«, 'geschissenes Garagentor`, ’g’schissene Kaffeemaschine`.

Skatologismen als Kosewörter treten in Verbindung mit meliorativen Attributen auf: ıkleiner Scheißer`, >mein süßer Scheißer « u.a.

Im Sprechakt >Beschimpfung können nicht nur pejorative Substantive, sondern auch Adjektive und Partizipien zum Einsatz kommen ( $\curvearrowright$ Du bist einfach nur scheiße‘; >die Situation ist beschissen`).

\subsection{Fiktive Beschimpfungen}

Dem Gebrauch skatologischer Pejorativa kann auch scherzhaft-kosende oder laudative Intention zugrunde liegen, z.B. wenn sie durch meliorative Suffixe, Attribute oder Possessivpronomen in Kosewörter umgewandelt werden und sich an Kinder richten: > Mein Scheißerl!<; >Oascherl! ‘; /Kleines Scheißerchen! 
Das unten angeführte Beispiel veranschaulicht nicht nur den laudativen Gebrauch des Lexems `Arschloch`, sondern auch dessen Wahrnehmung seitens des Adressaten: Als ein Journalist versucht hatte, Jazz Gitti, die Teilnehmerin des Projektes `Dancing Stars`, durch eine Behauptung auf den Arm zu nehmen, beschimpfte sie ihn:

Geh sch..., du Oar...loch, geh sch... Wenn du nicht für mich anrufst, zwick' ich dich in die Eier! Auf die Frage nach seiner Reaktion (ÖSTERREICH: Sie hat Sie Oar... loch geschimpft), antwortete der Journalist: KULIS: (lacht) Ja. Aber mit einem Smiley dabei. Es war in einer Mischung aus Erleichterung, Ärger und auch Respekt gesagt. ${ }^{26}$

Der Gebrauch von Pejorativa im Freundeskreis kann ein Zeichen enger Beziehung sein und signalisieren: »Unsere Freundschaft ist so stark und die Verbundenheit so eng, dass sie das Spiel mit den sonst beleidigenden Worten verkraften kann.«Die befragten Personen führen viele Beispiele für diesen Gebrauch an, auf den sie in den beigefügten Kommentaren hinweisen, z.B.:

`Geh scheißen, Häuseltschick - liebevoll beim Herumblödeln mit Freudinnen

>Drecksau < - scherzhaft zum Kollegen, der beim Arbeiten stört

>Du bist wirklich ein Oaschloch! oder >Du Trottel! $<$ - scherzhaft zum Freund, der z.B. gegenteiliger Meinung ist

>Arschloch $<$ - gegenseitig als Scherz zum Freund

Scherzhaft in einer Rede oder Begrüßung: ১Seavas, du Oasch!^

Ein interessantes Beispiel stellt `theatralisches Schimpfen « dar - wie von einer Befragten beschrieben: »Um Stress nach der Arbeit abzubauen, gebrauchen mein Mann und ich gegenseitig Beschimpfungen, proletenhaftes Benehmen nachahmend, z.B. Du Arsch! Leck mich am Arsch! u.a.«

\subsection{Selbstbeschimpfung}

Pejorativa, die zur Selbstbeschimpfung gebraucht werden, stellen fast ausschließlich die Klugheit oder die Intelligenz der sprechenden Person in Frage (`Ich Trottel/Idiot/Dummkopf!^) und funktionieren als universale Schimpfwörter in Situationen, in denen man durch eine unbedachte Handlung sich selbst Unannehmlichkeiten oder Schaden zugefügt hat. Dabei können auch skatologische aggressive Aufforderungen ( $>$ Leck mich am Arsch! $<$ - wenn ich was falsch gemacht habe) oder vulgäre Äußerungen ( $>$ Da kann i mi in Oasch beißen! - - wenn ich mit meinem Handeln unzufrieden bin) gebraucht werden.

Personenbezogene Schimpfwörter aus dem skatologischen Bereich werden zur Selbstbeschimpfung selten gewählt; es geht um Situationen, in denen bewusst oder unbewusst jemandem Schaden zugefügt wurde ist und 
dies bereut wird (`Arschloch! Ich bin grauslich!<; >Ich habe mich genau so verhalten wie ein Arsch! $)$.

Im Gegensatz zum seltenen Gebrauch von Skatologismen zur Selbstbeschimpfung in realen Konfliktsituationen, finden sich in literarischen Texten kunstvolle Beispiele der Selbsterniedrigung: "Nur ich bin die Scheiße einer zukünftigen Scheiße, die mich herausscheißt, bevor sie selber eine Scheiße in einem Menschenbauch wird «. ${ }^{27}$

\subsection{Abwertende Bemerkungen und Vergleiche}

Neben dem Gebrauch von skatologischen Schimpfwörtern kann die Beschimpfung auch mit abwertenden Vergleichen ( $>$ Du bist unnötiger wie a Wimmerl am Hintern`; ‘schierch, hässlich, ekelhaft, billig ... ıwie eine Scheiße / ein Arschloch $`$ ) und Bemerkungen erfolgen. Bei den abwertenden Bemerkungen treten Skatologismen entweder in direkter Bedeutung ( $>$ Die hat einen Arsch wie ein Pferd $`$ ) oder - häufiger - in übertragener Bedeutung als idiomatische Redewendungen auf:

>Du bist zu blöd / zu deppert zum Scheißen.

>Dem/Dir ham's ins Hirn g'schiss'n. <; >Dem hat wer ins Hirn g'schiss'n und ned obeloss'n.< 'geschissen ins Hirn'; >I glaub dir haben's ins Hirn g'schissen.

>Man könnte deinen Kopf mit dem Arsch verwechseln.<

>Der g'hert (mit der aidsverseuchten Brunnenröhre) in Oasch g'fickt.<

Abwertende Bemerkungen können auch in Form von Fragesätzen erfolgen (`Ham’s dir ins Hirn gschissen?<; >Was ist das für ein Scheiß?<; >Was soll der Scheiß? ).

Einige Bemerkungen sind durch ihre indirekte Form besonders heimtückisch: >Ich wusste gar nicht, dass man Scheiße so hoch stapeln kann.«; ,Weißt du noch, als ich meinen Arsch und du dein Gesicht aus dem Fenster hielten und alle meinten wir wären Zwillinge?^

\section{Sprechakt `Fluch`}

Im Gegensatz zum Sprechakt >Beschimpfung` wie auch zu den anderen aggressiven Sprechakten, ist der Sprechakt `Fluch nicht auf eine Person, sondern auf eine Situation gerichtet, die von der fluchenden Person selbst (oder - seltener - von jemandem anderen) verursacht wurde. Beim Fluchen werden im Deutschen meistens die skatologischen Wörter und Wendungen gebraucht: >Scheiße! ‘; >So a Schaß! ‘; >Scheiß! ‘; >Mist! <; >Dreck! ‘; >Scheißdreck!‘ 
Der Sprechakt >Fluch kann sowohl in Form einzelner Fluchworte (die selbständigen Ausrufesätze bilden), als auch in Form von (idiomatischen) Fluchwendungen vorkommen: >Zum Scheißen! <; >So eine Scheißerei!<; >Sowas Geschissenes! ; > Schas mit Quasteln! ‘ Flüche treten oft auch in Verbindung mit Attributen auf: heilige, verdammte, verfluchte, verfickte, fette, grüne Scheißer. Das Attribut kann auch nachgestellt werden: ১Scheißdreck, verdammter!‘; >Mist, verdammter! ‘ Typische syntaktische Konstruktion für den Sprechakt >Fluch`stellen Fluchwortketten dar: `Himmel(herrgott)(Arsch und Zwirn)!‘; >Himmel Arsch Herrgottssackra! ‘; >Himmel - Arsch - Verflucht!

Kennzeichnende Eigenschaft der Fluchworte ist ihre Ambivalenz, weshalb sie sowohl zur Äußerung negativer, als auch positiver Emotionen gebraucht werden können: >Himmel, Arsch und Wolkenbruch, das schmeckt aber verdammt gut!

Auch in emotional schwach geladenen Situationen wird geflucht: >Ach du Scheiße! - als Reaktion auf die Mitteilung von einem Missgeschick; s Scheiße, gib Antwort! - - als Äußerung der Ungeduld. Auf den routinierten Gebrauch in schwach emotional geladenen Situationen weisen Erzählberichte in literarischen Texten hin - wie etwa das >sagt ( und nicht etwa ১brüllt $<$ oder >schreit`) im nächsten Beispiel: » Scheiße`, sagt er. $^{28}$

Expletiver Gebrauch von Fluchwörtern (als Pausenfüller, um dem Gespräch einen Rhythmus zu verleihen), der im Englischen wie auch in den slawischen Sprachen häufig vorkommt, ist im Deutschen selten. In der Umgangssprache wäre die Verwendung des Ausrufes `oida` zu erwähnen; in der Belletristik ${ }^{29}$ findet sich das Beispiel `Scheiß mich an` (das in der Umgangssprache übrigens häufiger als Ausdruck des Unglaubens verwendet wird: ১Scheiß mich an is der deppert!^):

Ich frag nur, weil ich nicht dort gewesen bin. Ich hab nämlich wollen, aber scheiß mich an, zweiteilen kann ich mich nicht. ${ }^{30}$

Aber da hört er noch wie der Taxler sagt: »Ich frag die Wirtin: Scheiß mich an, wieso bist du denn heute so blass wie ein gekotztes Grießkoch? « ${ }^{31}$

»Scheiß mich an, das wird dir noch einmal leid tun«, sagt der gemütliche Chauffeur. ${ }^{32}$

Zum häufigsten Fluchwort \Scheißeく berichten ältere Interviewte, dass dieses Wort früher tabu war bzw. »von ganz primitiven Leuten aus der Unterschicht « verwendet wurde; erst in den letzten 30 Jahren hat es in Österreich dank deutscher Filme starke Verbreitung gefunden und ist >salonfähig` 
geworden. Tatsächlich kommt dieses Lexem in den Fragebögen der über 80-jährigen Personen nicht vor und die 50-jährigen Befragten erinnern sich an die Strafmaßnahmen, mit denen der Gebrauch dieses Wortes in ihrer Kindheit belegt war: >Als ich 16 war, habe ich für dieses Wort eine Ohrfeige bekommen und anderen damals erging es auch so $;>$ Im Alter von 8-9 Jahren hat mir das Wort Scheiße eine Watsch'n von meinem Vater eingebracht‘.

\section{Sprechakt \Drohung}

Skatologismen im Sprechakt >Drohung treten vor allem in irrealen Drohungen auf, deren Realisierung unvorstellbar erscheint und mit denen die drohende Person sich sowohl von negativen Emotionen befreit, als auch in scherzhafter Absicht in Szene setzt und ihre sprachliche Kreativität demonstrieren kann. Mit diesem Ziel können neben den einfachen ( $\gg I$ reiß da den Arsch auf!^) vor allem die erweiterten Drohungen gebraucht werden. Die Erweiterung erfolgt entweder als Erklärung der angedrohten Handlung ( $>I$ beiß da a Wendeltrepp'n in Oasch damit sich die Schaaß net dastessn! ২) oder als bildhafte hyperbolisierte Beschreibung der Folgen einer angedrohten Handlung:

>I reiß da di Brust und scheiß da aufs Herz!^

>I prack da ane, dass'd mitm Arsch auf'd Uhr schaust!

>I reiß da den Schädel ob und scheiß da in den Hals!

>I hau da ane, dass d mit'm Oasch auf' d Uhr schaust!

»Waunst nit glei die Pappn hoitst, daun hau i da a Gwind in Oarsch, daß’t Schraubn scheißt! $^{33}$

»I drisch da ane, dass da d'Händ beim Oasch Klavia spün! « ${ }^{34}$

Die Kommentare der Befragten zu den angegebenen Drohungen weisen auf deren scherzhaften Gebrauch hin. Scherzhaft bzw. ambivalent können die Drohungen auch Kindern gegenüber gebraucht werden: >Ich beiß dir in Popo / in den Hintern!

\section{Sprechakt /Verwünschung}

Mit diesem Sprechakt wird auf den Adressaten/die Adressatin oder auf sein/ihr Hab und Gut bzw. ihm/ihr nahe stehende Menschen ein Unheil beschworen. Im Deutschen werden Verwünschungen selten gebraucht; auch 
hier dominiert, wie bei den skatologischen Drohungen, die scherzhafte Intention. Die im Wienerischen häufige und meistens scherzhaft gebrauchte Verwünschung > Wünsche dir Krätze (Wimmel) am Oasch und zu kurze Hände zum Kratzen` zeigt Parallelen zum Jiddischen, das auf diesem Gebiet »eine geradezu dichterische Kultur $\aleph^{35}$ entwickelt hat (vgl. berühmte jiddische Verwünschung, die viele Sprachen in wörtlicher Übersetzung übernommen haben: >Alle Zähne sollen dir ausfallen bis auf einen - fürs Zahnweh!^). Das Kennzeichen dieses Typs von Verwünschungen ist die Verstärkung des Unheilwunsches im zweiten Teil: >Der Blitz (Kugelblitz) soll dich treffen! $<->$ Der Blitz (Kugelblitz) soll dich beim Scheißen treffen!

Die Verwünschung kann auch mit paralinguistischen Mitteln, z.B. Gesten, begleitet werden: >Am Oasch so große Warzen (zeigen) und so kurze (zeigen) Hände zum Kratzen (scherzhaft).

\section{Sprechakt >Aufforderung}

Im Gegensatz zur Verwünschung sind aggressive Aufforderungen im modernen Deutsch häufig. Am häufigsten wird mit ihrem Gebrauch zum Verschwinden (`Schleich di! $)$ und zum Schweigen (`Halt die Gosch(e)n!^) aufgefordert. Skatologismen treten in metaphorischen Aufforderungen auf, die die Form von festen Redewendungen haben und sowohl zu einer Handlung auffordern (`Beweg jetzt deinen fetten (faulen) Arsch $`$ ) als auch zur Abweisung der Adressatin / des Adressaten dienen können:

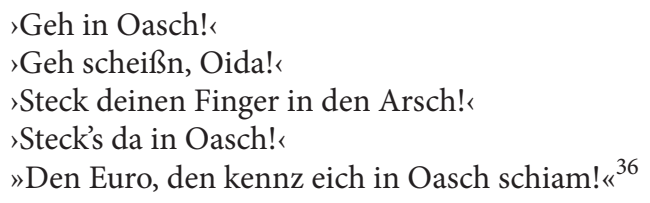

Die Aufforderung >Leck mich am Arsch` wird euphemistisch Götz-Zitat genannt - nach der bekanntesten (aber nicht der ältesten, denn dieselbe Aufforderung finden wir auch in Grimmelshausens Simplicius Simplicissimus) Erwähnung, nämlich in Goethes Götz von Berlichingen, wo sie noch mit einer anderen Präposition vorkommt - >im Arsch ‘. Diese Aufforderung kann in Verbindung mit Modaladverbien (`kreuzweise $`$ ) oder in elliptischer Konstruktion (`Du kannst mich kreuzweise ) auftreten. Dieselbe Aufforderung kann auch euphemistische Formen annehmen (siehe dazu auch unten, Abschnitt 9): 
>Du kannst mir den Buckel hinunterrutschen!

>Du kannst mich am Buckel küssen!

>Du kannst mi buglfünferln!

>Besuche mich im Mondschein!

Die Aufforderungen funktionieren auch in erweiterter Form: `Geh in Oasch - da hast du's nicht weit! ‘ oder: 'Geh in Oasch, wann in Hümme kummst eh net! «; >Leck mich doch bis in Oasch eine und zwoa glei links!

Infolge des häufigen Gebrauchs lässt sich bei den aggressiven Aufforderungen ein Prozess der Ökonomisierung beobachten: etwa, wenn die Aufforderung von Interviewten infolge der Zusammenrückung als ein Wort angegeben wird (`Gehscheißen! «, >Leckdomihypodezent! «) oder durch das Auslassen von Personal- bzw. Possessivpronomen, Präpositionen oder Verben (`Leck Arsch!^).

Neben der Aufforderung zur konkreten Handlung, dem Abreagieren negativer Emotionen oder der generellen Abweisung werden die aggressiven Aufforderungen auch mit dem Ziel gebraucht, ein Gespräch bzw. eine Konfliktsituation endgültig abzubrechen, eine als Zumutung empfundene Bitte zurückzuweisen oder zu provozieren (`Scheiß di net an! $)$. Die aggressiven Aufforderungen können, wie das empirische Material zeigt, neben den negativen auch die positiven Emotionen und Empfindungen $(>\mathrm{Du}$, leck mi (am Oasch), des Schnitzel war gut! ‘ oder `Du leck mi am Oasch, der Film war gut!^) sowie Überraschung (`Ja, da leckst mich doch am Arsch!^) zum Ausdruck bringen.

Häufig kommt es zum scherzhaften Gebrauch aggressiver Aufforderungen, auf den die Kommentare der Befragten hinweisen:

'Geh scheißen` (scherzhaft-abweisend im Freundeskreis)

>Leck mich doch (am Oasch)< (auch scherzhaft im Freundeskreis um das Gespräch zu beenden)

`Geh ma net am Oasch`(auch scherzhaft)

\section{Gegen-Aufforderungen und andere Reaktionen auf aggressive Sprechakte}

Eine verbreitete Reaktion auf eine Aufforderung ist, wie auch bei den anderen aggressiven Sprechakten, die >Retourkutsche $<$ - das `Zurückschlagen mit demselben Schimpfwort / derselben Aufforderung: >Leck mich am Arsch! $<>$ Du mich auch!

Häufig werden von den befragten Wienerinnen und Wienern scherzhaft-ironische Fragen oder Antworten als Reaktionen auf aggressive Sprech- 
akte angegeben (typisch für den Kommunikationsstil >Wiener Schmäh $`$ ), z.B. als Reaktion auf die Aufforderung >Leck mich am Arsch! $>$ : Reinigend oder zum Genuss?^ oder: >Hängen Sie ihn (den Arsch) an die Wand und machen Sie’s selbst! ‘ In einer Konfliktsituation können diese Reaktionen die Situation entschärfen, indem die angreifende Person durch die scherzhafte verbale Reaktion `sprachlos` (schmähstad) wird oder (wenn der Angreifer/ die Angreiferin ebenfalls die Kunst des Schmähführens beherrscht und mit schlagfertigen Antworten reagiert) sich im kreativen Wortkampf auflösen und das Publikum aufheitern. Dies geht etwa aus der Erzählung einer Interviewten hervor, die auf die brutale Aufforderung eines Fahrgastes in der Straßenbahn - »Leck mich am Arsch! - antwortet: »Danke für das Angebot, aber ich tue es nicht bei jedem!«, was zur Aufheiterung der Fahrgäste und einer Entschärfung der Spannung führt. Eine ähnliche Reaktion stammt von einem Lehrer, der auf die Äußerung eines schwierigen und aggressiven Schülers - »Leck mich am Arsch!« - ruhig antwortet: »Ich mag das nicht, ich krieg dann Sodbrennen « und damit Begeisterung der Schülerinnen und Schüler für sein cooles Verhalten erntet.

Raffiniert sind Reaktionen, die auf Verwirrungstechniken beruhen, etwa wenn die aggressive Aufforderung in die höfliche Rede eingebaut wird und dadurch noch stärker zur Geltung kommt: Ein Befragter berichtete, er habe auf die Beschimpfung eines anderen Autofahrers mit »Finden Sie nicht, dass die Art, wie Sie mit mir reden, Sie unter Ihrem Wert verkauft?« geantwortet und nach einer Pause hinzugefügt: »Und gehen Sie scheißen!«

In einem ähnlichen Kontext, in Verbindung mit der gehobenen Äußerung >Habe die Ehre`, was der ganzen Aussage eine sarkastische Färbung verleiht, gebraucht eine vulgäre Aufforderung auch jene literarische Figur, die als Inbegriff vulgärer Sprechweise gelten kann - Edmund Sackbauer (Mundl): »Habe die Ehre und leckt's mich am Arsch! « ${ }^{37}$

\section{Euphemismen im skatologischen Bereich}

Als Euphemismen treten mildere oder verschleiernde Wörter und Ersatz-

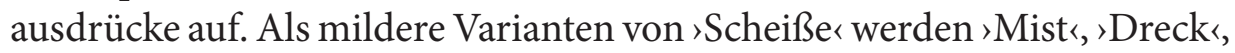
>Kacke/Gacke/Gacki` gebraucht, statt `Geh (doch) scheißen!‘ kann >Geh (doch) kacken!^verwendet werden. 
Bei den verschleiernden Euphemismen unterscheide ich nach Kiener ${ }^{38}$ zwei Arten: 1. Euphemismen, die nach Bedeutungsähnlichkeit, und 2. Euphemismen, die nach Lautähnlichkeit zum Tabuwort gebildet werden. Zur ersten Art gehört auch der Gebrauch fremdsprachiger Pejorativa (engl. shit, frz. merde). Bei den pejorativen Wörtern und Ausdrücken ist der zweite Weg der Euphemisierung (durch Lautähnlichkeit) verbreitet. Dabei gibt es verschiedene Möglichkeiten:

a) Reduktion des Tabuwortes auf Null, die als nicht steigerbare Form des Euphemismus gilt (Nulleuphemismus): >Du kannst mich (mal)! (statt: >Du kannst mich (mal) am Arsch lecken! «) oder >Leck mich... (Auslassung von ram Arsch $<$ );

b) Reduktion auf die Anfangslaute (in den Fragebögen werden die Anfangsbuchstaben angegeben): >Sch...< (anstatt: >Scheiße < bzw. >Schaaß<); >So ein Sch...! ( (statt: >So ein Scheiß!/Schaaß! ), >LmaA (statt: >Leck mich am Arsch $<$ ); >Scheipi< (euphemistischer Okkasionalismus: abgekürzt aus `scheiß und `Piefke`);

c) Substitution - Umstellung oder Ersatz zweier oder mehrerer Laute: z.B. in den Fragebögen statt `Scheiße $<->$ Scheibe $<$ (

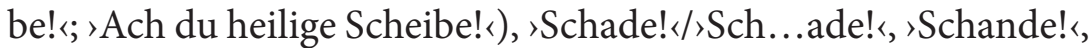

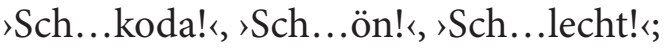

d) Substitution + Erweiterung mit neutralen oder kosenden Lexemen: >Sch...önes Wetter!‘, >Scheibenkleister!‘, >Scheibenhonig!‘ (statt: >Scheiße!`), >Geh sch...au dir das Wetter an!^ (statt: >Geh scheißen! ), >Leck mich am Aaaaaarm! «, >Leck doch die Tante am Ohr! (statt: >Leck mich am Arsch!`).

Durch Substitution können auch individuelle euphemistische Aufforderungen gebildet werden: >Geh a Schüssal beißen! ( (statt: >Geh a bissl scheißen! ) und `Sche geißen! ( (statt: >Geh scheißen! ২). - Einen interessanten Fall der okkasionellen Euphemisierung stellt die Verbindung mit einem Meliorativum in Form einer Zusammenrückung dar: >Scheißeschatzi! ( (so flucht laut einer Befragten ihre Freundin aus einer adeligen Familie). - Auf der syntaktischen Ebene ist ein euphemistischer Vorgang interessant, der der Litotes verwandt ist und eine Umkehrung des Gemeinten darstellt: >Du kannst mich gern haben!` als Euphemismus der Aufforderung >Leck mich am Arsch!^. Einen Prozess, der der Euphemisierung entgegengesetzt ist, stellt der Gebrauch von skatologischen Dysphemismen dar, die vulgäre Bezeichnun- 
gen für neutrale, alltägliche, nicht tabuisierte Sachverhalte, Handlungen, Gegenstände sind, z.B `Schasvagoder für den Ventilator im Wienerischen oder >etwas verkacken` (in der Bedeutung >etwas schlecht machen`, >etwas vermasseln`) in der Jugendsprache.

\section{Attraktion von Skatologismen}

Ein weiteres Charakteristikum des Funktionsmechanismus pejorativer Lexik und aggressiver Sprechakte ist ihre Attraktion (Anhäufung): Der schimpfenden Person fehlt es im Affektzustand oft an treffenden Worten, weshalb sich ihre Erregung in der Mehrfachnennung zeigt. Die Attraktion beobachten wir auf verschiedenen Ebenen - als Anhäufung pejorativer Halbaffixe (`Drecks-Scheißtag`, ,Scheißkackendreck`, >Hurenscheißdreck`), einzelner Pejorativa oder aggressiver Sprechakte. Die graphische Wiedergabe erfolgt auch durch Zusammenrückung:

১Oidamotherfuckingscheiße!

»leckdumichamarsch ich muß raus da « ${ }^{39}$

»leck-du-mich-am-arsch-du-hammel ${ }^{40}$

»scheißoaschdreeggfaschissanefafiggde! « ${ }^{41}$ (als die letzte U-Bahn vor der Nase wegfährt)

Die Attraktion aggressiver Sprechakte erscheint in verschiedenen Kombinationen:

- Beschimpfung - Emotionsthematisierung - Beschimpfung: >Du Geschissener, du gehst ma am Oasch, du geschissenes Arschloch du!

- $\quad$ aggressive Aufforderung - aggressive Aufforderung - Beschimpfung: >Geh scheißen! Na dann geh scheißen du Arschloch!

- aggressive Aufforderung - Beschimpfung: »Jetzt verstopf dir doch endlich selber dein blödes Maul, du impotentes Stück Scheiße. ${ }^{42}$

\section{Ausblick}

Das pejorative Vokabular des Deutschen ist in der fäkal-analen Sphäre verankert, das bedeutet, dass die Skatologismen über den Wörtern und Ausdrücken aus dem sexuellen oder gotteslästerlichen Bereich dominieren. 
Diese Besonderheit beobachten wir von der morphologisch-semantischen (z.B. in Form skatologischer Halbaffixe oder als metaphorische und metonymische Pejorativa aus dem fäkal-analen Bereich) bis zur syntaktischen Ebene (beleidigende Bemerkungen und Vergleiche, Emotions- und Situationsthematisierungen, Fluchwortketten, Verwünschungen, Drohungen oder aggressive Aufforderungen). Das bestätigt die Hypothese von der Zugehörigkeit der deutschen Sprache hinsichtlich der Verankerung ihres pejorativen Vokabulars zur >fäkal-analen Schimpfkultur ${ }^{43}$

Es kommt auch zur Kreuzung von Pejorativa aus der fäkal-analen und der sexuellen Sphäre: sowohl auf der morphologisch-semantischen Ebene (in Teilen zusammengesetzter Lexeme) als auch im Kontext, z.B. als Verbindung mit den Attributen ( $>$ Du verficktes Scheißding! $)$ ) oder als Attraktion aggressiver Sprechakte (>Fick dich, Scheißoaschloch!^).

\section{Literaturverzeichnis}

\section{Sekundärliteratur}

Aman, Reinhold: Die klügsten Beschimpfungen findet man im Jiddischen. "Psychologie heute« 11 (1996), S. 32-35.

Dundes, Alan: Sie mich auch! Das Hinter-Gründige in der deutschen Psyche. Weinheim, Basel: Beltz 1985.

Gauger, Hans-Martin: Das Feuchte und das Schmutzige. Kleine Linguistik der vulgären Sprache. München: C.H. Beck 2012.

Girtler, Roland: Der Strich. Erotik der Straße. Wien: Edition 1994.

Havryliv, Oksana: Pejorative Lexik. Untersuchungen zu ihrem semantischen und kommunikativ-pragmatischen Aspekt am Beispiel moderner deutschsprachiger, besonders österreichischer Literatur. Frankfurt/M. u.a.: Peter Lang 2003.

Havryliv, Oksana: Verbale Aggression. Formen und Funktionen am Beispiel des Wienerischen. Frankfurt/M. u.a.: Peter Lang 2009.

Havryliv, Oksana: Verbale Aggression: das Spektrum der Funktionen. »Linguistik Online« 82.3 (2017) (Sprache und Gewalt / Language and Violence. Hg. Aneta Stojić), S. 27-48.

Jay, Timothy: Why We Curse. A neuro-psycho-social theory of speech. Philadelphia: Benjamins 2000.

Karl, Daniel Walter: Das nicht immer so goldene Wienerherz. Das besondere Schimpfwörterlexikon mit ausgewählten 1300 Spitzenausdrücken. Wien: K. W. Daniel 2006.

Kiener, Franz: Das Wort als Waffe. Zur Psychologie der verbalen Aggression. Göttingen: Vandenhoeck \& Ruprecht 1983.

43 Aman: Die klügsten Beschimpfungen; Dundes: Sie mich auch!; Gauger: Das Feuchte und das Schmutzige; Kiener: Das Wort als Waffe: Stavyc'ka: Украӥнська мова без табу; Zhelvis: Поле брани. 
Searle, John R: Intentionalität. Eine Abhandlung zur Philosophie des Geistes. Übers. Harvey P. Gavagai. Frankfurt/M.: Suhrkamp 1991.

Stavyc'ka, Lesja = Ставицька, Леся: Українська мова без табу. Словник нецензурої лексики та ї̈ відповідників. Обсиенізми. Евфемізми. Сексуалізми. Київ: Критика 2008.

Zhelvis, Vladimir I. = Жельвис, Владимир И.: Поле брани: сквернословие как социальная проблема в языках и культурах мира. Москва: Ладомир 2001.

\section{Literarische Quellen}

Artmann, Hans Carl: How much, schatzi? Frankfurt/M.: Suhrkamp 1973.

Awadalla, El: SEAWAS, BIST A KRANK? Tiefe und tiefgründige Dialoge im Krankenhaus. Wien: Milena 2014.

Bauer, Wolfgang: Magic Afternoon. In: ders.: 3 Stücke. München: Deutscher Taschenbuch Verlag 1969, S. 7-45.

Bernhard, Thomas: Claus Peymann verlässt Bochum und geht als Burgtheaterdirektor nach Wien. In: ders.: Werke. Hgg. Martin Huber, Wendelin Schmidt-Dengler. Frankfurt/ M.: Suhrkamp 2012, S. 61-75.

Haas, Wolf: Auferstehung der Toten. Hamburg: Hoffmann und Campe 2006.

Hinterberger, Ernst: Mundl. Ein echter Wiener geht nicht unter. Wien: Deuticke 1995.

Kehlmann, Daniel: F. Reinbek/H.: Rowohlt 2013.

Schwab, Werner: Troilluswahn und Cressidatheater. In: ders.: Dramen III. Graz, Wien: Droschl 1994, S. 7-74.

Schwab, Werner: Der Himmel mein Lieb meine sterbende Beute. In: ders.: Fäkaliendramen. Graz, Wien: Droschl 1992, S. 193-236.

Schwab, Werner: Übergewicht, unwichtig: UNFORM. In: ders.: Fäkaliendramen. Graz, Wien: Droschl 1993, S. 59-120.

Schwab, Werner: Faust:: Mein Brustkorb: Mein Helm. In: ders.: Dramen III. Graz-Wien: Droschl 1994, S. 75-134. 